\title{
Numerical investigation of flow regimes in T-shaped micromixers: benchmark between finite volume and spectral element methods
}

\author{
Chiara Galletti, , Alessandro Mariotti, Lorenzo Siconolfi, Roberto Mauri \\ and Elisabetta Brunazzi \\ Dipartimento di Ingegneria Civile e Industriale, University of Pisa, Pisa, \\ Italy
}

May 31, 2018

\section{Abstract}

Computational Fluid Dynamics (CFD) is very appealing to investigate mixing and reaction in microdevices, as it allows easily investigating different operating conditions as well as mixer geometries. This latter aspect is very important as the flow in microdevices is laminar so the mixing between reactants should be promoted by a clever mixer design, aimed at breaking the flow symmetries. Recently time periodic motions, that improve mixing, have been observed to take place in a T-junction at low Reynolds numbers. In this case the numerical modelling should be based on Direct Numerical Simulations (DNS), thus involving high computational resources. In this work, two different CFD approaches, i.e. finite volume and spectral element methods, are applied and compared for the analysis of the mixing process in the well known T-shaped micromixer. Spectral elements methods are particularly suited for DNS, however they have been scarcely applied to study micromixers, while plenty of works can be found with finite volume methods. The analysis is carried out using both ideal and non-ideal liquid binary mixtures, the latter presenting a negative fluidity of mixing (i.e., the viscosity of the mixture is higher than that of the pure components). Moreover the numerical results are validated with simple flow visualization experiments.

\section{Keywords}

Flow visualization; Microreactor; T-junction; Computational Fluid Dynamics; Direct Numerical Simulations

* corresponding author. Prof. Chiara Galletti, Dipartimento di Ingegneria Civile e Industriale, University of Pisa, Largo L. Lazzarino 2, I-56122 Pisa, Italy. email chiara.galletti@unipi.it 


\section{Introduction}

Microdevices, consisting of multiple sub-millimeter channels in which fluids flow continuously and chemical reactions take place, are very attractive for process intensification. The small reaction volume, well controlled residence time and large heat transfer rate (due to the large surface to volume ratio) enable reactions to be carried out under less dilute conditions, resulting in higher yields than those that can be obtained with conventional batch reactors. Indeed, it has been shown that microreactors may be particularly advantageous for several reactions of pharmaceutical ${ }^{1}$ and fine chemistry processes ${ }^{2}$, as well as for green chemistry, due to their potential of reducing waste of material and energy.

Due to the small dimensions involved, the flow in micromixers and microreactors is laminar, so mixing should be ensured through a special design of the microdevice, mainly aimed at breaking the flow symmetries and promoting mixing by splitting and recombination of the flow streams. Hence, large attention is paid to the optimization of the geometrical configuration of the microchannels, as well as to the operating conditions that should be adopted to improve the mixing process.

The experimental analysis of mixing and reaction in microdevices calls for advances techniques, such as micro Laser Induced Fluorescence (micro-LIF) [3 [5 micro Particle Image Velocimetry (micro-PIV) ${ }^{3}$ that allow measuring the concentration and the flow field, respectively. A comprehensive review on these experimental techniques is provided by Sinton ${ }^{[6]}$. Due to the tiny dimensions of the investigated volume, special arrangements should be adopted. For instance in comparison with classical PIV, micro-PIV requires the use of very small particles (i.e., with $100 \div 300 \mathrm{~nm}$ diameter) which should also be fluorescent. Another difference with classical techniques, is that it is not possible to use a light sheet to investigate the flow or concentration fields in one plane, whereby the complete microchannel volume is illuminated. Hence, the 3D information is obtained by collecting the emitted light only from the focus plane through, for example, confocal laser scanning microscopy. Very recently, Riccomi et al. 7 applied in a T-junction a novel technique, the Ghost Particle Velocimetry (GPV), showing that it has the potential to 
become, if further developed, a reliable and economical 3D flow visualization technique for micro/milli-fluidic sized applications. GPV uses a bright field illumination and, as $\mathrm{PIV}$, is based on imaging and cross-correlating small tracers displacements $\underline{8}$. Here the distinctive feature is the use of the speckle pattern produced by high refractive index nanoparticles.

In this framework, the design of microreactors could benefit of Computational Fluid Dynamics (CFD), as it allows easily investigating different geometrical configurations and operating conditions without any scale issue, hence providing large cost and time savings. Indeed, lots of numerical investigations of micromixers and microreactors can be found in literature, some of them for instance aimed at the geometrical optimization of the mixer (e.g., 910 11 12$]$ ). One of the most studied geometry is the T-shaped micromixer, in which the inlets join perpendicularly the mixing channel. Such geometry has been largely investigated numerically, highlighting the presence of both steady (stratified,

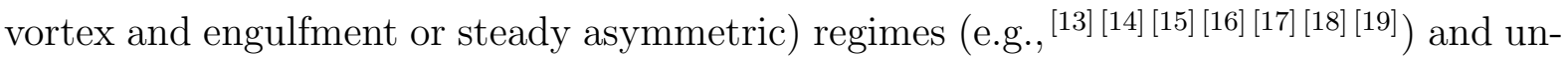
steady (asymmetric and symmetric time-periodic) regimes (e.g., 202 212 22] ). In particular the unsteady regimes were firstly discovered numerically [14 [20] and only subsequently they were observed experimentally $\stackrel{23}{23}$. Moreover, numerical predictions may be used to shed light into the mechanisms driving flow instabilities using stability analysis techniques 24$] 25]$.

Numerical simulations are computationally expensive especially in case of unsteady regimes that require the use of Direct Numerical Simulations (DNS). Moreover, there is debate on the grid resolution that should be adopted to accurately predict the concentration field especially in case of chemical reactions $\stackrel{26}{2}$.

Most of the above numerical investigations are performed with commercial CFD codes, based on finite volume methods. However, the use of spectral elements codes, that have been particularly conceived for high computational performance as that required in DNS simulations, could be very appealing for the numerical analysis of micromixers and microreactors.

Hence, the present work is aimed at comparing the performance of two different CFD 
Figure 1: Sketch of the T-shaped micromixer (not in scale).

approaches, i.e finite volume and spectral element methods, for the analysis of the mixing process in the well known T-shaped micromixer. This analysis is carried out using both ideal and non-ideal liquid binary mixtures, the latter presenting a negative fluidity of mixing (i.e., the viscosity of the mixture is higher than that of the pure components). Moreover we want to show how CFD can be used to help interpreting and gaining information from easy and cheap experiments, that have been performed for the ideal mixture.

\section{Test case}

The mixer is a T-junction, shown in Figure 1 along with the adopted reference frame. The inlet channels have a square cross-section, with $W_{i}=H=1 \mathrm{~mm}$, while the mixing channel presents a $2: 1$ aspect ratio, i.e. $W_{o}=2 H=2 \mathrm{~mm}$, so that its hydraulic diameter is $d=4 H / 3$. The length of the inlet channels, $L_{i}=40 \mathrm{~mm} \approx 30 d$, is enough to ensure a fully developed flow at the confluence, while the mixing channel length, $L_{o}=60 \mathrm{~mm}$ $\approx 45 d$, allows the complete evolution of vortical structures 21 .

The mixer is fed with liquids with equal flow rates. The density of the liquids is the same, i.e. $\rho(\phi)=\rho$, while the viscosity $\mu$ is a function of composition. In particular, two different cases are considered:

- case $A$ ideal binary liquid mixture, whose viscosity is constant upon composition, i.e.

$$
\mu(\phi)=\mu_{0} ;
$$

- case $B$ non-ideal binary liquid mixture, whose viscosity depends on composition, through a parabolic function, i.e.

$$
\mu(\phi)=\mu_{0}[1+8 \phi(1-\phi)] .
$$


Figure 2: Non-dimensional viscosity $\mu / \mu_{0}$ as function of mixture composition $\phi$.

Figure 3: Scheme illustrating the methodology of the work.

In the above equation $\phi$ represents the mass or volume fraction of one liquid in the mixture. For case $\mathrm{B}$ the maximum viscosity is $\mu=3 \mu_{0}$ at $50 \%$ of composition $(\phi=0.5)$; this behavior is very similar to that of the water-ethanol mixture which is often employed in microfluidics 27 28] 17. The viscosity-composition diagrams for case A and case B are shown in Figure 2 .

\section{Methodology}

The present work is aimed at comparing the performance of two different CFD approaches in predicting the flow and concentration fields in micromixers. In addition to the simulations performed with the two codes, experimental flow visualizations are also performed and compared with numerical results. The overall methodology is depicted in the scheme of Figure 3 .

\subsection{Experimental set-up and image-analysis procedure}

The micromixer is made of polymethylmethacrylate (PMMA) and thus it is fully transparent. The experiments regard case A, hence clear deionized water was fed in one mixer inlet channel and deionized water dyed by Ponceau 4R (Sigma-Aldrich) in the other. Due to the low concentration of $C_{0}=1.9 \cdot 10^{-3} \mathrm{~mol} / \mathrm{L}$ of the dye in water any influence on the fluid behavior can be neglected. The mass flow was provided by a KD Scientific syringe pump. The experimental set-up comprised an upright microscope (model Eclipse 80i from Nikon, Japan) equipped with a D-LH 12V-100W halogen lamp as light source. A magnifying lens of $4 \times$ and an aperture value setting equal to $N . A .=0.13$ were used to acquire the images by using the high-speed camera Optomotive Velociraptor HS.

To avoid aliasing problems at higher Reynolds number, the sampling frequency and the resolution were set at 387 frames/s and $920 \times 2048$ pixels, respectively. In this way, the 
exposure time of each image is equal to $\delta T=10^{-6} \mathrm{~s}$, thus three order of magnitude smaller than the sampling frequency. As the light illuminates the complete microchannel, the recorded light intensity is averaged along the optical path through the fluid and provides a depth-averaged concentration field. Preliminary calibration runs showed that the Lambert-Beer's linear relationship between light absorbance and dye concentration holds also with our experimental set-up and the chosen operating conditions. Hence, the acquired images were processed with a fully automatize and off-line procedure with Matlabß to convert recorded light intensity images into normalized dye concentration images according to the following equation, applied for each $(i, j)$-th pixel of the images:

$$
\frac{\ln \left(\operatorname{Iblank}_{i j}\right)-\ln \left(I_{i j}\right)}{\ln \left(\operatorname{Iblank}_{i j}\right)-\ln \left(\operatorname{Imax}_{i j}\right)}=\frac{C_{i j}}{C_{0}}
$$

where, $I_{i j}$ and $C_{i j}$ are the instantaneous values of light intensity and dye concentration, respectively, while, $\operatorname{Iblank}_{i j}$ and $\operatorname{Imax}_{i j}$ are the reference values, i.e. the light intensity values recorded when the mixer is full of the blank and the $C_{0}$ dye solution. The experimental procedure required thus, for each set of flow visualization experiments, the acquirement first of the references, obtained by feeding the mixer only with the blank and the $C_{0}$ dye solution, respectively, before using the two streams together. In order to limit the uncertainty related to time fluctuation of pixel intensity, the reference $\operatorname{Iblank}_{i j}$ and $\operatorname{Imax}_{i j}$ are chosen to be their arithmetic mean values over 100 frames each. Details on the calibration and image analysis procedures can be found in Mariotti et al. 29], together with a more detailed description of the whole experimental set-up.

\section{Direct Numerical Simulations}

\subsection{Governing equations}

The fluid motion is completely described by the velocity field $u$, the modified pressure $P$ and the volume/mass fraction $\phi$, which satisfy the following continuity, Navier-Stokes 
and convection-diffusion equations, here in their non-dimensional form:

$$
\begin{gathered}
\nabla \cdot \mathbf{u}=0 \\
\frac{\partial \mathbf{u}}{\partial t}+\mathbf{u} \cdot \nabla \mathbf{u}=-\nabla P+\frac{1}{R e}\left[\nabla \cdot \frac{\mu(\phi)}{\mu_{0}}\left(\nabla \mathbf{u}+\nabla \mathbf{u}^{T}\right)\right] \\
\frac{\partial \phi}{\partial t}+\mathbf{u} \cdot \nabla \phi=\frac{1}{P e} \nabla^{2} \phi .
\end{gathered}
$$

Lengths, velocities and time are scaled using the mixing channel hydraulic diameter, $d$, the average fluid velocity $U$ (that is the same in the mixing and inlet channels) and convective time $d / U$, respectively. Hence $R e=\frac{\rho U d}{\mu_{0}}$ and $P e=\frac{U d}{D}=S c R e$ are the Reynolds and Peclet numbers, respectively, with $S c=\frac{\mu_{0}}{\rho D}$ denoting the Schmidt number. In the above equations, the density $\rho$ is constant, while the viscosity $\frac{\mu(\phi)}{\mu_{0}}$ is 1 for case $\mathrm{A}$ or it is estimated through Equation 2 for case B.

\subsection{Numerical solvers}

As mentioned in the Introduction, two different approaches were compared by using the

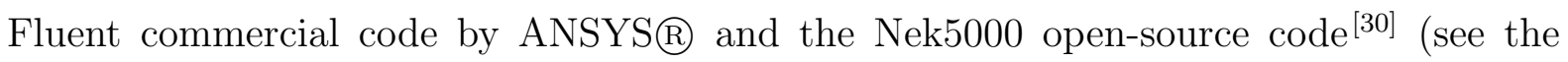
scheme of Figure 3).

Fluent is based on finite volume methods; the spatial discretization of the T-mixer consists in a fully structured grid with $4.7 \mathrm{M}$ cells. The cells are cubical at the confluence region, while they elongate along the inlet and the outlet channels. In particular, in a cross-section of the mixing channel there are $40 \times 80$ cells. A grid independency study indicated that a coarser grid (i.e., with $20 \times 40$ cells in the mixing channel cross-section) could

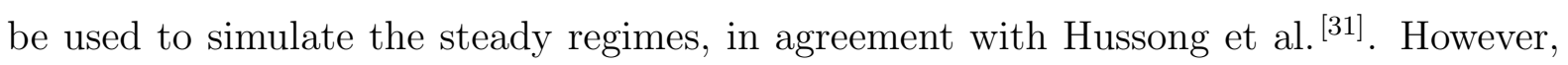
such grid was found to be unable to describe the unsteady dynamics at large Reynolds numbers. A transport equation for a user defined scalar was added; the diffusivity of the scalar was that of the dye used in the experiments, corresponding to a Schmidt number $S c=5000$. The concentration field was hence discretized using the same mesh size as for the velocity field; an extended discussion to justify this assumption was presented in 


\section{Galletti et al. 32}

In the steady regimes, a steady solver with a second order discretization scheme was employed, with a SIMPLE algorithm to handle the pressure-velocity coupling. At convergence the normalized residuals for velocities did not change with iterations and most of the times fell below $1 \cdot 10^{-11[16]}$. The steadiness of the solution was also checked by monitoring velocity and concentration values in some locations in the mixing channel at different iterations. In the unsteady regimes, a transient solver with a second order implicit advancement method was employed using a time step corresponding to a CFL number of $\approx 5$. Typically such runs were carried out using 32 cores and required several days of computations. A $\mathrm{C}++$ subroutine was implemented in the code to allow the visualization of the vortical structures.

Nek5000 is based on the spectral element method and it is massively parallelized. The spatial discretization of the T-mixer consists of a structured grid with 12250 hexahedral elements, with $5 \times 10$ elements in the mixing channel cross-section, with functions being interpolated through $N$ th-order Lagrange polynomial interpolants, based on tensorproduct arrays of Gauss-Lobatto-Legendre (GLL) quadrature points in each spectral element. The polynomial order $N$ for the velocity is fixed at 7 , while an order 5 is imposed for the pressure, thus using the common $P_{N} / P_{N-2}$ scheme. Time discretization was handled with a third order backward differential formula (BDF3). The diffusive terms are treated implicitly, while a third order explicit extrapolation (EXT3) is considered for the convective terms. The explicit extrapolation for the convective terms in the BDF3-EXT3 scheme enforces a small time step, corresponding to a CFL number $\leq 0.3$. A scalar equation was added to predict the dye concentration; for this equation, stability issues forced to use a rather low $S c$ number, i.s. $S c=O(100)$ as in Fani et al. 25 . The unsteady simulations of both Fluent and Nek5000 were run in order to capture at least 20 periodic cycles after discarding the initial transient. As for the computational cost, the two codes behaved similarly; indeed, even though Nek5000 is computationally very efficient, it uses an explicit method which requires small time steps. In particular, about 2000 and 5000 CPU hours were needed to characterize steady and unsteady, respectively, regimes with 
Nek5000, when running on 72 cores (2.30 GHz Intel cluster). Instead, Fluent required about 1500 and $4000 \mathrm{CPU}$ hours for steady and unsteady, respectively, regimes, when running on 24 cores (2.3 GHz AMD Opteron workstation). However, it is difficult to give a precise indication of computational costs; for instance, the simulations of the steady engulfment regimes are usually quick, but they can become extremely slow near the critical Re numbers, i.e. near the transition points to different regimes. Nevertheless, Nek5000 is expected to outperform Fluent in case of more complex micromixer geometries requiring heavy computational grids, because of its high scalability allowing a massive parallelization. Moreover, Nek5000 is an open-source code, hence it can be run using an arbitrary number of parallel cores without any license issue. The difference between the two codes are summarized in Table 1 .

As for the boundary conditions, in both codes no-slip velocity and no-mass-flux are set at the channel walls, while uniform velocity and concentration is imposed at the entrance, as the inlet channels are long enough to provide a fully developed flow at the $\mathrm{T}$ confluence. A pressure outlet condition with ambient pressure is given at the exit in the Fluent

simulations, while free outflow conditions (i.e., $\frac{\partial u_{x}}{\partial y}=0, P-\frac{1}{R e} \frac{\partial u_{y}}{\partial y}=0$ and $\frac{\partial u_{z}}{\partial y}=0$ ) are set in the Nek5000 simulations.

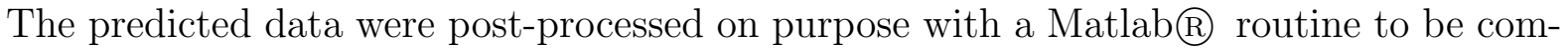
pared with the flow visualizations. Since the experimental (2D) images result from the light attenuation through the fluid, depending on the dye concentration, the numerical (3D) concentration distribution in the mixer is averaged over the mixer depth (i.e., along the $z$ coordinate). This is shown in the scheme of Figure 3 . This procedure allows to use simple flow visualization experiments for a quantitative validation of the numerical models.

\section{Results}

The benchmark between the two numerical approaches will be discussed in the following text. First, the results for the ideal binary mixture, i.e. case A, are presented in detail, 
Table 1: Details of Fluent and Nek5000 set-up.

\begin{tabular}{|c|c|c|}
\hline & Fluent & Nek5000 \\
\hline Method & finite volume method & $\begin{array}{c}\text { spectral element } \\
\text { method }\end{array}$ \\
\hline characteristics & commercial code & $\begin{array}{l}\text { massively parallel } \\
\text { open-source code }\end{array}$ \\
\hline discretization & $\begin{array}{c}4.7 \mathrm{M} \text { cells }(40 \times 80 \text { in } \\
\text { mixing channel } \\
\text { cross-section })\end{array}$ & $\begin{array}{l}12250 \text { spectral } \\
\text { elements }(5 \times 10 \text { in } \\
\text { mixing channel } \\
\text { cross-section })\end{array}$ \\
\hline interpolation & second order upwind & $\begin{array}{l}N \text {-th order Lagrange } \\
\text { polynomial } \\
\text { interpolants }(N=7 \\
\text { for velocity, } N=5 \text { for } \\
\text { pressure) with Gauss- } \\
\text { Lobatto-Legendre } \\
\text { (GLL) quadrature } \\
\text { points }\end{array}$ \\
\hline $\begin{array}{c}\text { temporal } \\
\text { advancement }\end{array}$ & $\begin{array}{l}\text { second order implicit } \\
\text { method }\end{array}$ & $\begin{array}{l}\text { third order backward } \\
\text { differential formula } \\
\text { for diffusive terms, } \\
\text { third order explicit } \\
\text { extrapolation for } \\
\text { convective terms }\end{array}$ \\
\hline CFL & $<5$ & $<0.3$ \\
\hline Schmidt number & $S c=5000$ & $S c=O(100)$ \\
\hline cores & 24 & 72 \\
\hline
\end{tabular}


here in comparison with flow visualizations provided by a dedicated experimental activity. Then, the results for the non-ideal binary mixture, i.e. case B, are discussed. In the following, the local dye concentration is normalized by the concentration of the inlet stream, hence the concentration values range from 0 to 1 in the mixer. Moreover, all coordinates in the following figures and graphs are made non-dimensional by using the mixing channel hydraulic diameter, i.e. $X=\frac{x}{d}, Y=\frac{y}{d}$ and $Z=\frac{z}{d}$.

\subsection{Case A}

Figure 4a,b,c compares the experimental flow visualization at $R e=160$ and the depthaveraged dye concentration maps obtained with Fluent and Nek5000 for the ideal mixture. Although the pattern is complex, the agreement at first glance is very satisfactory. In the same figure (i.e., Figure 4 d,e), the vortical structures estimated from the flow fields predicted with Fluent and Nek5000 are visualized through the $\lambda_{2}$-criterion ${ }^{33}$. This criterion identifies a vortex as a connected fluid region where the second largest eigenvalue of the symmetric tensor $\mathbf{L}=\mathbf{S} \cdot \mathbf{S}+\mathbf{A} \cdot \mathbf{A}$, is negative. $\mathbf{S}$ and $\mathbf{A}$ are the symmetric and anti-symmetric part of the velocity gradient, i.e. the strain rate and vorticity tensors, respectively $\nabla \mathbf{U}=\mathbf{S}+\mathbf{A}$. There is a very good agreement between the $\lambda_{2}$ iso-surfaces predicted with the two codes. Such vortical structures are those typical of the engulfment regime that, for the T-mixer aspect ratio investigated here, occurs when $140<R e<220^{21}$. The engulfment regime is characterized by a tilting in the $x-z$ plane of the recirculation bubbles generated at the confluence of the streams at the top of the T-mixer 24 . Two counter-rotating vortical structures stem from these recirculation regions near the top walls of the mixer, and these vortices are convected into the mixing channel, forming two legs for each vortical structure. However, due to the tilting of the separation bubbles, each of the two vortices originated in the bubble is asymmetric; this leads to a couple of weak legs, that soon disappear in the mixing channel and a couple of strong legs.

A signature of these complex vorticity structures (Figure $4 \mathrm{~d}$,e) can be identified in the experimental flow visualization (Figure $4 \mathrm{a}$ ) and the predicted depth-averaged concentra- 
(dia)

Figure 4: Experimental dye concentration field (a) and those predicted with (b) Fluent and (c) Nek5000. Isosurface of the $\lambda_{2}$ vortex indicator obtained with (d) Fluent and (e) Nek5000. Case A, $R e=160$.

tion fields (Figure $4 \mathrm{~b}, \mathrm{c}$ ). For instance we can recognize the recirculation bubbles at the top of the T-junction, although such bubbles appear superimposed in the 2D images due to their tilting. The white and black regions in the center of the mixer stem from the presence of the two weak legs, whereas the gray regions that expand moving down the mixing channel are due to the mixing induced by the strong legs.

Figure 5 a,b shows the dye distribution, predicted with Fluent and Nek5000 in the mixing channel cross-section at $Y=-0.15$. The corresponding $y$-vorticity and in-plane velocity vectors are reported in Figure 6a,b, thus indicating the presence of a pair of strong counter-rotating vortices and a pair of weak ones, corresponding to the strong and weak legs, respectively. Looking at the dye distribution in Figure 5 , some minor differences can be observed between the two codes. For instance, Fluent predicts some dark region near the l.h.s. (and white region near the r.h.s.), that comes from the presence of the two strong legs that pushed the incoming stream towards the opposite side; whereas such region is scarcely visible in the Nek5000 simulations. The quantitative comparison between experimental depth-averaged dye concentration and those predicted with the two codes is shown in Figure 5r, that reports profiles along the $x$ axis in the mixing channel cross-section at $Y=-0.15$. The graph indicates a very good matching, with some minor differences near the walls, that may be partly imputed to the intrinsic limits of the experimental technique very close to the walls. In particular, the experimental profile is not exactly antisymmetric (e.g., the $\phi$ value at $X=-0.75$ is different from the value at $X=0.75)$. As for the flow field, the agreement between the two codes is very satisfactory as shown in Figure 6a,b for both $y$-vorticity and in-plane velocity. We can observe how the codes uses different nodal locations to store the velocity values; these are uniformly distributed across the mixing channel cross-section in Fluent, unlike Nek5000 where the 
Figure 5: Distribution of dye concentration in the mixing channel cross-section at $Y=$ -0.15 predicted with (a) Fluent and (b) Nek5000. (c) Profiles along the $X$ axis at $Y=-0.15$ of average dye concentration obtained with the two codes. Case A, Re $=160$.

(ald $)$

Figure 6: Distribution of $Y$-vorticity and in-plane velocity vectors in the mixing channel cross-section at $Y=-0.15$ predicted with (a) Fluent and (b) Nek5000. (c) Profiles along the $X$ axis at $Y=-0.15$ and $Z=0$ of $Y$ velocity component obtained with the two codes. Case A, $R e=160$.

distribution is not uniform. The profile of the $y$-velocity along the $x$ direction at $Z=0$ (i..e, mid-plane) and $Y=-0.15$ is reported in Figure 6c that again indicates a nearly perfect matching between the two predictions.

The degree of mixing can be defined using the bulk or cup mixing average mass fraction. Accordingly, we can estimate the variance of the flow-weighted concentration, $\sigma_{b}$, as:

$$
\sigma_{b}^{2}(y)=\frac{1}{N \bar{u}} \sum_{i}\left(\phi_{i}-\bar{\phi}_{b}\right)^{2} u_{i} ; \quad \overline{\phi_{b}}=\frac{\sum_{i} \phi_{i} u_{i}}{N \bar{u}}
$$

where $\bar{\phi}_{b}$ is the bulk, or cup mixing average, mass fraction, $u_{i}$ is the axial velocity of the mixture at point $i$ within the cross-section, while $\bar{u}=\sum_{i} u_{i} / N$ is its mean value, i.e., the ratio between the volumetric flux and the cross-section area. Hence, the degree of mixing $\delta_{m}$ on a specific cross-section can be defined as:

$$
\delta_{m}=1-\frac{\sigma_{b}}{\sigma_{\max }} ; \quad \sigma_{\max }=\sqrt{\bar{\phi}_{b}\left(1-\bar{\phi}_{b}\right)}
$$

where $\sigma_{\max }$ is the maximum value of the standard deviation $\sigma$, that is achieved when the two inlet streams remain completely segregated.

The degree of mixing predicted by the two numerical codes along the mixing channel for $R e=160$ is reported in Figure 7. Both codes predict a sharp increase of the degree of mixing due to the strong transverse convection in the confluence region, up to $|Y| \approx 4$, although $\delta_{m}$ estimated by Fluent is slightly larger than that predicted by Nek5000. This 
Figure 7: Degree of mixing along the mixing channel predicted with Fluent and Nek5000. Case $\mathrm{A}, R e=160$.

is due to the numerical diffusion induced by the finite grain size of the numerical grid, typical of all finite volume methods. As we proceed down the mixing channel, the degree of mixing predicted with Nek5000 continues to increase, whereas that estimated with Fluent tends to reach a nearly asymptotic value. This behavior is due to the fact that, while in the Fluent simulations we have adopted a physically sound Schmidt number, $S c=5000$, corresponding to the ratio between the water kinematic viscosity and the molecular diffusivity of the dye, in the Nek5000 simulations we have assumed a much smaller value, i.e., $S c=P e / R e=62.5$ for $P e=10000$, due to the fact that larger $S c$ results into numerical instability. A simplified analysis can be made by assuming a uniform flow. In such case, the diffusion length in the transversal direction can be found as:

$$
\delta \approx \sqrt{D \frac{y}{U}} \rightarrow \frac{\delta}{d} \approx \sqrt{\frac{Y}{P e}} .
$$

According to the above formula, when $Y \approx 4$ the diffusion length $\delta_{N e k}$ predicted by Nek5000 (hence using $P e=S c R e=10000$ ) is approximately equal to the Fluent grid size (i.e., $\approx d / 100$ ). Hence, at this axial coordinate the degrees of mixing predicted by the two codes will coincide, in agreement with Figure 7. However, as we proceed down the mixing channel, the increment of the diffusion length predicted by Nek5000 $\delta_{N e k}$ leads to a large increase of the degree of mixing. Instead, the diffusion length estimated by Fluent is much smaller, as $\frac{\delta_{\text {Fluent }}}{\delta_{\text {Nek }}}=\sqrt{\frac{S c_{\text {Nek }}}{S c_{\text {Fluent }}}} \approx \frac{1}{10}$, leading to a smooth increase of the degree of mixing along the channel.

Figure 8 and Figure 11 show experimental images and depth-averaged concentrations predicted with Fluent and Nek5000, along with vortical structures as identified through the $\lambda_{2}$-criterion for two instants $\theta$ at $R e=280$. At such Reynolds number, the flow exhibits an unsteady time-periodic behavior with a Strouhal number $S t=\frac{d}{U \tau}=0.218$, where $\tau$ is the characteristic time of the periodic motion. The vortical structures are typical of the unsteady asymmetric regime ${ }^{21}$ that occurs for $220<R e<320$. 
(di)

Figure 8: Experimental dye concentration field (a) and those predicted with (b) Fluent and (c) Nek5000. Isosurface of the $\lambda_{2}$ vortex indicator obtained with (d) Fluent and (e) Nek5000. Case A, $R e=280$ and $\theta / \tau=0$.

Figure 9: Experimental dye concentration field (a) and those predicted with (b) Fluent and (c) Nek5000. Isosurface of the $\lambda_{2}$ vortex indicator obtained with (d) Fluent and (e) Nek5000. Case A, Re $=280$ and $\theta / \tau=0.5$.

In particular, at the instant $\theta / \tau=0$ shown in Figure 8, the vortical structures resemble those of the engulfment (i.e., steady asymmetric) regime, even though the vortical structures at the top of the mixer are more tilted and closer, and the downstream part of the vortical legs is more swirled than in the steady engulfment regime. A signature of such swirling is well visible in the experimental image (Figure 8a). The agreement between the experimental snapshot and the predicted depth-averaged concentration is also in this case very good for both codes. It is worth noting, as discussed above, that the small $S c$ used in Nek5000 does not affect results in such region that extends up to $|Y|=3$. The vortical structures computed from the flow field with the two codes look very alike also in the small details (see Figure $8 \mathrm{~d}, \mathrm{e}$ ). Same conclusions can be drawn for the snapshots at the instant $\theta / \tau=0.5$ shown in Figure 11. Here the vortical structures are merging at $Y \approx-1.5$, and this will lead to the formation of a vorticity blob that will be convected through the mixing channel. The passage of this blob causes the twisting and deformation of the legs of the vortices. In the mean time, new vortical structures form in the top part of the mixer, thus leading to a new flow cycle. Such complex dynamic is better explained in Andreussi et al. 21. Again the agreement between the two codes in the prediction of the depth-averaged concentration and the vortical structures is very

high. A quantitative comparison with the measurements is provided in Figure 10 and Figure 11 for $\theta / \tau=0$ and $\theta / \tau=0.5$, respectively. Here experimental depth-averaged 
Figure 10: Distribution of dye concentration in the mixing channel cross-section at $Y=$ -1.3 predicted with (a) Fluent and (b) Nek5000. (c) Profiles along the $X$ axis at $Y=$ -1.3 of average dye concentration obtained with the two codes. Case A, $R e=280$ and $\theta / \tau=0$.

Figure 11: Distribution of dye concentration in the mixing channel cross-section at $Y=$ -1.3 predicted with (a) Fluent and (b) Nek5000. (c) Profiles along the $X$ axis at $Y=$ -1.3 of average dye concentration obtained with the two codes. Case A, $R e=280$ and $\theta / \tau=0.5$.

concentration profiles along the $x$ coordinate are compared to those predicted with Fluent and Nek5000 at $Z=0$ and $Y=-1.3$. For sake of clarity also the distribution of dye concentration in the same mixing channel cross-sections, predicted with the two codes, is reported.

At both instants the agreement between the two codes and the experiments is satisfactory. Again, even though the $S c$ number used in the Nek5000 simulations is much smaller than the real one, at such location the diffusion length is still low due to the short residence time, thus not significantly impacting on the concentration field.

\subsection{Case B}

The two codes are now compared for the non-ideal binary mixture showing a viscosity dependent on composition, characterized by a negative fluidity of mixing, being the viscosity at $50 \%$ of composition three times larger than that of the pure components. In such case the formation of a viscous layer hampers the mixing process; in particular the engulfment regime is not characterized by a sharp increase of the degree of mixing, as for the ideal mixture, but a gradual increase is observed. Figure 12 compares depth-averaged concentrations and vortical structures predicted with Fluent and Nek5000 at $R e=400$, which is still in the steady (engulfment) regime. Indeed the vortical structures appear similar to those observed at $R e=160$ for case A, yet presenting a couple of strong and a couple of weak legs. For the non-ideal mixture the steady regime persists up to $R e=550$ 
Figure 12: Experimental dye concentration field (a) and those predicted with (b) Fluent and (c) Nek5000. Isosurface of the $\lambda_{2}$ vortex indicator obtained with (d) Fluent and (e) Nek5000. Case B, $R e=400$.

Figure 13: Distribution of dye concentration in the mixing channel cross-section at $Y=$ -1.3 predicted with (a) Fluent and (b) Nek5000. (c) Profiles along the $X$ axis at $Y=$ -1.3 of average dye concentration obtained with the two codes. Case B, $R e=400$.

(instead of $R e=220$ for case A) ${ }^{222}$.

The two codes are well in agreement, as proven also by the dye distribution in the mixing channel cross-section at $Y=-1.3$ (Figure 13). The quantitative comparison is provided as profiles along the $x$ coordinate of the depth-averaged concentration (Figure 13) c.

The distribution of $y$-vorticity and in-plane velocity vectors predicted with the two codes in the mixing channel cross-section at $Y=-1.3$ are reported in Figure 14 . Here the presence of two strong co-rotating vortices is well evident. The agreement between the two codes may be also appreciated by the profiles of the $y$-velocity along the $x$ direction at $Z=0$ (mid-plane) reported in Figure 6c; some minor differences can be observed.

For case $\mathrm{B}$, the unsteady regime is found to take place at $550<R e<700^{22}$, instead of at $220<R e<510$ for case A. Figures 15,18 compare depth-averaged concentration distribution and vortical structures predicted with Fluent and Nek5000 at different instants at $R e=600$. At $\theta / \tau=0$. the flow morphology is similar to that of the engulfment regime with the tilted recirculation structures at the top of the mixer and the couples of

Figure 14: Distribution of $Y$-vorticity and in-plane velocity vectors in the mixing channel cross-section at $Y=-1.3$ predicted with (a) Fluent and (b) Nek5000. (c) Profiles along the $X$ axis at $Y=-1.3$ and $Z=0$ of $Y$ velocity component obtained with the two codes. Case $\mathrm{B}, R e=400$. 
Figure 15: Dye concentration field (a) and those predicted with (b) Fluent and (c) Nek5000. Isosurface of the $\lambda_{2}$ vortex indicator obtained with (d) Fluent and (e) Nek5000. Case $\mathrm{B}, R e=600$ and $\theta / \tau=0$.

Figure 16: Dye concentration field (a) and those predicted with (b) Fluent and (c) Nek5000. Isosurface of the $\lambda_{2}$ vortex indicator obtained with (d) Fluent and (e) Nek5000. Case $\mathrm{B}, R e=600$ and $\theta / \tau=0.125$.

strong and weak legs in the mixing channel. However, the flow does not show a perfect point-central symmetry in each mixing channel cross-section as in case A. This is more evident in the following instant $\theta / \tau=0.125$ at which the vortical structures in the channel tend to merge forming a vorticity blob, that is well evident at $\theta / \tau=0.25$ (Figure 17). Such vorticity blob is convected through the mixing channel, as its passage deforms the legs of the vortical structures. However, in comparison with the dynamics of the unsteady asymmetric regime in case A (see Figures 8, 9), the vortical legs here appear more bent towards one side, i.e. the r.h.s at $\theta / \tau=0.375$. This is due to the lost of the central-point symmetry in each mixing channel cross-section. Such behavior is also well evident from the depth-averaged concentration fields, the 2D maps looking no more symmetric as for case A. In the next half period (from $\theta / \tau=0.5$ to $\theta / \tau=1$ ) a similar dynamic occurs but the legs are bent towards the l.h.s.. Hence, in each period, the blob of vorticity forms by the merging of the vortical legs and is convected bending firstly the flow structures, on, say, the r.h.s.; subsequently new vorticity generates at the top of the mixer and the merging of the legs generate a blob of vorticity that bends the legs on other side, i.e. the l.h.s.. 
Figure 17: Dye concentration field (a) and those predicted with (b) Fluent and (c) Nek5000. Isosurface of the $\lambda_{2}$ vortex indicator obtained with (d) Fluent and (e) Nek5000. Case $\mathrm{B}, R e=600$ and $\theta / \tau=0.25$.

Figure 18: Dye concentration field (a) and those predicted with (b) Fluent and (c) Nek5000. Isosurface of the $\lambda_{2}$ vortex indicator obtained with (d) Fluent and (e) Nek5000. Case $\mathrm{B}, R e=600$ and $\theta / \tau=0.375$.

\section{Conclusions}

Direct Numerical Simulations of a T-shaped micromixer have been performed with both finite volume and spectral elements methods, using, respectively, Fluent and Nek5000. The latter code is particularly suited for Direct Numerical Simulations, due to the high degree of parallelization. Results have shown that the agreement between the codes in predicting the flow field is very high, provided that finite volume simulations are performed with a sufficiently fine grid and suited interpolation.

The analysis of the concentration field showed a good matching in the confluence region, where traversal motion occurs. Here, a comparison with simple flows visualization experiments, in case of an ideal mixture, was made by estimating the depth-averaged concentration to obtain 2D maps, to be examined against experimental images. The agreement was very satisfactory for both steady and unsteady time-periodic regimes. With further moving along the mixing channel, the concentration fields predicted with the two codes differ significantly. Indeed the degree of mixing estimated with Nek5000 shows a steeper increase along the channel than that obtained with Fluent. This behavior is imputed to the larger diffusivity, due to the small Schmidt number of typically $S c \approx 100$, employed in the Nek5000 simulations to avoid numerical instabilities; hence the passive scalar is no more a tracer as the dye used in the experiments. For this reason, a poor prediction of 
the concentration field may be expected with Nek5000 for many applications involving liquid mixing. This could be for example the case of some water-organic liquid mixtures used for producing drug-carrier nanoparticles through solvent displacement techniques. For instance ethanol in water exhibits $S c \approx 1000$. Instead, acetone in water has $S c \approx 80$, for which Nek5000 should work reasonably well. It is however worth nothing that, despite in Fluent simulations a physically sound $S c$ number was used, results are affected by the numerical diffusion induced by the finite grain size of the numerical grid. As for the numerical discretization, the second order upwind employed in this work has been reported to provide similar results to third order methods in T-shaped micromixer for

similar $R e^{34}$. Instead, spectral element methods exhibit very little numerical diffusion and dissipation $[30$.

Similar conclusions on the agreement between the two codes were also drawn in the case of a non-ideal binary mixture presenting a negative fluidity of mixing. Here the presence of a viscous layer at the confluence hampers the mixing process, so the engulfment is delayed and it is not accompanied by a sharp increase of the degree of mixing. The unsteady regime takes place also in this case, although for larger Re with respect to the ideal mixture case. Moreover the dynamics was found to be characterized by the lost of the central-point symmetry in each mixing channel cross-sections; as a consequence, the vortical legs in the mixing channel are bent toward one side for half period of the periodic motion and toward the other side for the next following period.

\section{Acknowledgments}

This work was supported by the University of Pisa through the "Progetti di Ricerca di Ateneo PRA 2017-2018" funding program. The authors wish also to thank CINECA computing center (Bologna, Italy) for the computational resources under ISCRA programs (class B project "MIRE"). Moreover the authors are grateful to Matteo Antognoli, Tommaso Andreussi, Deborah Spaltro, Tommaso Pannuzi and Cesare Merello. 


\section{References}

[1] D. M. Roberge, L. Ducry, N. Bieler, P. Cretton, B. Zimmermann, Chem. Eng. Technol. $2005,28,318$.

[2] I. Rossetti, M. Compagnoni, Chem. Eng. J. 2016, 296, 56.

[3] N. Kockmann, T. Kiefer, M. Engler, P. Woias, Sensor. Actuat. B-Chem. 2006, 117, 495.

[4] N. Ait Mouheb, D. Malsch, A. Montillet, C. Solliec, T. Henkel, Chem. Eng. Sci. $2012,68,278$.

[5] W. Wang, S. Zhao, T. Shao, Y. Jin, Y. Cheng, Chem. Eng. J. 2012, 192, 252.

[6] D. Sinton, Microfluid. Nanofluid. 2004, 1, 2.

[7] M. Riccomi, F. Alberini, E. Brunazzi, D. Vigolo, Chem. Eng. Res. Des 2018, 133, 183.

[8] S. Buzzaccaro, E. Secchi, R. Piazza, Phys. Rev. Lett. 2013, 111, 48101.

[9] M. Roudgar, E. Brunazzi, C. Galletti, R. Mauri, Chem. Eng. Technol. 2012, 35, 1291.

[10] L. Siconolfi, A. Fani, S. Camarri, M. V. Salvetti, Europ. J. Mech. B/Fluids 2016, 55, 360.

[11] J. Ortega-Casanova, Chem. Eng. Process. 2017, 117, 18.

[12] P. Hermann, J. Timmermann, M. Hoffmann, M. Schluter, C. Hofmann, P. Lob, D. Ziegenbalg, Chem. Eng. J. 2018, 334, 1996.

[13] M. Engler, N. Kockmann, T. Kiefer, P. Woias, Chem. Eng. J. 2004, 101, 315.

[14] D. Bothe, C. Stemich, H-J Warnecke, Chem. Eng. Sci. 2006, 61, 2950.

[15] A. Soleymani, H. Youse

, I. Turunen, Chem. Eng. Sci. 2008, 63, 5291. 
[16] C. Galletti, M. Roudgar, E. Brunazzi, R. Mauri, Chem. Eng. J. 2012, 185-186, 300.

[17] C. Galletti, G. Arcolini, E. Brunazzi, R. Mauri, Chem. Eng. Sci. 2015, 123, 300.

[18] A. V. Minakov, V. Y. Rudyak, A. A. Gavrilov, A. A. Dekterev, Thermophys. Aeromech. 2012, 19, 385.

[19] A. Lobasov, A. Minakov, Chem. Eng. Process. 2018, 124, 11.

[20] S. Dreher, N. Kockmann, P. Woias, Heat Transfer Eng. 2009, 30, 91.

[21] T. Andreussi, C. Galletti, R. Mauri, S. Camarri, M. V. Salvetti, Comput. Chem. Eng. 2015, 76, 150.

[22] C. Galletti, E. Brunazzi, R. Mauri, Chem. Eng. Sci. 2017, 164, 333.

[23] S. Thomas, T. Ameel, Exp. Fluids 2010, 49, 1231.

[24] A. Fani, S. Camarri, M. V. Salvetti, Phys. Fluids 2013, 25, 064102.

[25] A. Fani, S. Camarri, M. V. Salvetti, Phys. Fluids 2014, 26, 074101.

[26] D. Bothe, A. Lojewski, H.-J. Warnecke, Chem. Eng. Sci. 2011, 66, 6424.

[27] G. Orsi, M. Roudgar, E. Brunazzi, C. Galletti, R. Mauri, Chem. Eng. Sci. 2013, 95, 174.

[28] G. Orsi, C. Galletti, E. Brunazzi, R. Mauri, Chem. Eng. Trans. 2013, 32, 1471.

[29] A. Mariotti, C. Galletti, R. Mauri, M. V. Salvetti, E. Brunazzi, Chem. Eng. J. 2018, $341,414$.

[30] P. F. Fischer, J. W. Lottes, S. G. Kerkemeier, Web page: http://nek5000.mcs.anl.gov 2008.

[31] J. Hussong, R. Lindken, M. Pourquie, J. Westerweel. In: Ellero, M., et al. (Eds.), IUTAM Symposium on Advances in Micro- and Nano-fluidics. Springer, Heidelberg, 2009, 191. 
[32] C. Galletti, E. Brunazzi, R. Mauri, Chem. Eng. Trans. 2015, 43, 1645.

[33] J. Jeong, F. Hussain, J. Fluid Mech. 1995, 285, 69.

[34] N. Solehati, J. Bae, A. P. Sasmito, Comput. Fluids 2014, 96, 10.

[35] M.O. Deville, P.F. Fischer, E.H. Mund. High-order methods for incompressible fluid flow. Cambridge University Press, Cambridge, 2002. 